\title{
Sexuality Research within Neuroimaging: A Review of Progress toward Greater (?) Gender Equality and Sex Positivity
}

\author{
Ariana Tart-Zelvin, M.S. and Xiaomeng Xu, Ph.D. \\ Idaho State University
}

Scientific fascination with the brain's role in behavior has an extensive history. In 1937, Penfield and Boldrey used electrical probes to develop maps for sensory and motor cortices of the human male brain. These maps, commonly referred to as the cortical homunculus, are still used today - mostly unaltered. However, only male patients were used and thus, the maps only contain markers for male genitalia. This bias has continued into the present day with research focusing on healthy, typically heterosexual, males (Arnow et al., 2002; Ferretti et al., 2005; Holstege et al., 2003; Mouras et al., 2003; Safron et al., 2007) and predominantly overlooking research on female sexuality, particularly with a sex-positive framework. This gender bias has interfered with clinicians' knowledge of and ability to promote women's physical and mental sexual health. Fortunately, recent progress in neuroimaging research has resulted in greater gender equality within the field. These studies have resulted in important changes to newer versions of the Diagnostic and Statistical Manual of Mental Disorders (DSM-5).

\section{Early Neuroimaging Studies}

A review of early neuroimaging literature on sexuality contains many studies conducted on healthy males. A plethora of ethical and legal concerns were believed to exist regarding the participation of women in clinical studies (Mastroianni et al., 1994). Historically, a lack of knowledge regarding female reproductive processes and anatomy resulted in numerous regulations concerning their participation in research studies.

Concerns were expressed regarding women's ability to have children if they underwent clinical procedures or even exercised vigorously (Beresini, 2013). While pregnant women are still considered a vulnerable population, science does not support the idea that women are incredibly fragile and that running two miles will harm (?) the uterus. Recent studies do continue to exclude women for other reasons. For example, menstrual cycles greatly vary from woman to woman these hormonal differences can make results more difficult to interpret depending on the data (Moyer, 2010).

Arnow et al. (2002) examined sexual arousal in healthy heterosexual males. At that time, little research existed regarding the neural activation associated with sexual response. Although the researchers did not provide a reason for excluding females, they did mention that examining potential differences in brain activation between males and females would be a beneficial future direction for the research. Holstege et al. (2003) conducted a neuroimaging study that examined activation during healthy male 
ejaculation. Similar to Arnow et al. (2002)'s study, Mouras et al. (2003) examined the neural response of healthy heterosexual males to sexual stimuli.

In 2005, Ferretti et al. continued the male-focused research by examining sexual arousal in healthy heterosexual males, specifically neural activation during penile erection (as opposed to simple sexual arousal) and the timing of the activation. The authors mentioned that further studies are needed to better define neural activations associated with "human sexual behavior" (p. 9185) and pointed out that their findings hold important implications for clinical diagnosis, therapy, and treatment for sexual dysfunction, but failed to acknowledge that the implications only applied to healthy heterosexual males.

Safron et al. (2007) conducted a study to examine neural activations associated with sexual arousal in both heterosexual and homosexual males. The inclusion of homosexual males in this study increased the diversity of this field and the applicability of the study results.

The imaging studies conducted during this earlier period with females are far fewer than those including only males and focus on sexual dysfunction in some manner. This focus on sexual dysfunction promotes a more sex-negative message for the female results. Thus, our understanding of healthy sexual function in females has been severely limited.

However, in 2004, Basson conducted a neuroimaging study to advance the understanding of women's sexual function and dysfunction. This study provided some unexpected results including a poor correlation between women's subjective experience of arousal and activation in brain areas associated with the organization of reflexive genital vasocongestion. These results have since been added to newer models of sexual response for females and resulted in revisions of definitions of women's sexual dysfunction (Basson, 2004). For example, Basson improved an older model of sexual response by creating a new model of a "blended sexual response cycle reflecting spontaneous desire augmenting a cycle initiated for reasons other than desire" (p. 716).

In 2009, Arnow et al. utilized functional magnetic resonance imaging (fMRI) to investigate hypoactive sexual desire disorder (HSDD) in females, comparing 16 females with HSDD to 20 females with no history of HSDD. They used subjective measures of sexual arousal, peripheral sexual response via vaginal photoplethysmograph, and fMRI across three time points. Researchers concluded that compared to those without HSDD, females with HSDD encode arousing stimuli and retrieve past erotic experiences differently and allocate significantly more attention to monitoring their responses. More recent research has also shown that females with HSDD exhibit different limbic and cortical activation associated with the acquisition, encoding, and memory retrieval of information (Woodward, 2013) and greater activation in areas associated with a "heightened attention to one's own physical state" (p. 1068). 


\section{Recent Neuroimaging Studies}

Recent neuroimaging sex research studies are moving away from exclusively heteronormative male sexuality. In 2011, Komisaruk et al. utilized fMRI methodology to better understand the neural systems that underlie female sexual response. This study provided the first evidence that the genital sensory cortex mapped in men by Penfold and Boldrey in 1937 exists in women. Additionally, this study had a sex-positive approach, addressing sexual response in healthy women and examining a variety of behaviors including both imagining self-stimulation and actual self-stimulation of the vagina, clitoris, cervix, and nipples. The results showed that the genital sensory cortex was activated both by imagining and performing self-stimulation. It also demonstrated that stimulation of the nipples, typically not considered a genital area and not mapped as such by Penfield and Boldrey, resulted in genital sensory cortex activation.

Additional sex-positive studies have been conducted and have been presented at conferences or as part of a dissertation, but may not yet be formally published. This trend signals a continuation of a more sex-positive framework for the future of this field. For example, Wise (2014) conducted two studies as part of a dissertation, which helped elucidate the neural time-course of orgasm in healthy women (lead-up, during, and recovery periods). Wise showed neural orgasm response differences (lead-up) and similarities (during and recovery) between self-stimulation and partner-stimulation. Wise also clarified that although both imagined and actual self-stimulation are associated with genital sensory cortex activation, the imagined stimulation must be erotic for this effect to occur (e.g., imagining genital self-stimulation with a dildo will elicit activation but imagining stimulation with a speculum will not).

Similarly, Komisaruk et al. (2013) investigated how men's anatomy maps onto the sensory cortex in a more sex-positive and progressive manner, seeking to extend the original work of Penfield and Boldrey (1937) which only examined penile response. Komisaruk et al. (2013), investigated multiple regions including penile glans and shaft, scrotum, testicles, urethra, perineum, rectum, prostate, and nipples, and tested both mild and forceful self-stimulation. Their results showed that many of these areas activate the sensory cortex, although in different and distinguishable patterns, illustrating the diversity of sexual responses. Komisaruk et al. also demonstrate that men's responses during nipple self-stimulation were similar to those of women from the Komisaruk et al. (2011) study.

Finally, recent studies have provided us with a richer and broader understanding of sexuality. For example, Huynh, Willemsen, and Holstege (2013) have utilized neuroimaging (in this case positron emission tomography - PET) to better understand sexual pleasure (i.e., orgasms and ejaculation) in both men and women. They studied sexual pleasure in the context of other bodily changes and their evolutionarily important functions. They found that increased pituitary activation may indicate higher plasma 
concentrations of oxytocin and prolactin, which enhance sperm and egg transport and are involved in ovulation and vaginal and uterine movements.

\section{Clinical Implications and Future Directions}

The studies mentioned above inform clinicians' understanding and treatment of patients' sexual dysfunctions as presented in the DSM. Currently, clinicians utilize the DSM-5, which is a recent transition from the DSM-IV-TR. In the DSM-IV-TR and previous editions, sexual-related disorders were categorized under "Sexual and Gender Identity Disorders" and had not been revised since the 1980s. Gender identity disorder was added to the DSM-III in 1980. In 1987, sexual disorders in the DSM-III-R were meaningfully altered based on the extant literature. The category then remained fairly unaltered until 2013.

With the publication of the DSM-5 in 2013, new diagnoses were added and other existing diagnoses were separated, modified, or removed, highlighting the importance of research findings and improvements in research design inclusivity. One major change classified sexual disorders separately from gender dysphoria, the new term for gender identity disorder. Importantly, gender-specific sexual dysfunctions were added to the DSM-5 while the gender-neutral sexual dysfunction diagnosis was removed. Some of the other new diagnoses that were added include; delayed ejaculation, other specified sexual dysfunction, and genito-pelvic pain/penetration disorder. An example of an existing disorder that was modified includes changing female sexual arousal disorder to female sexual interest/arousal disorder implying that "interest" and "arousal" represent two separate and important components.

These changes to the DSM represent steps in the right direction, but there exists room for improvement regarding sexual-related studies and associated clinical diagnoses. For example, factors such as desire and arousability have scarcely been studied in healthy females or females who suffer from sexual dysfunction. Further, several current clinical sexual disorders list criteria that are more fitting for the heterosexual population. These criteria could be amended to better include individuals who do not identify as cisgender and/or heterosexual.

Although the history of sexuality neuroscience has not been particularly sexpositive, the future of this field is more promising as researchers focus on sexual response, rather than simply dysfunction, a diversity of participants, and a variety of sexual behaviors and pleasure responses. As this field continues, we anticipate the emergence of additional studies that help us to better understand and appreciate sexuality in all of its forms, which will contribute to more positive views of sexuality in and out of clinical contexts. 


\section{References}

Arnow, B. A., Desmond, J. E., Banner, L. L., Glover, G. H., Solomon, A., Lake Polan, M., Lue, T. F., \& Atlas, S. W. (2002). Brain activation and sexual arousal in healthy, heterosexual males. Brain, 125, 1014-1023.

Arnow, B. A., Millheiser, L., Garrett, A., Lake Polan, M., Glover, G. H., Hill, K. R., Lightbody, A., Watson, C., Banner, L., Smart, T., Buchanan, T., \& Desmond, J. E. (2009). Women with hypoactive sexual desire disorder compared to normal females: A functional magnetic resonance imaging study. Neuroscience, $158,484-502$.

Basson, R. (2004). Recent advances in women's sexual function and dysfunction. Menopause, 11 (6), 714-725.

Beresini, E. (2013, March 25). The myth of the falling uterus. Retrieved from http://www.outsideonline.com/1783996 /myth-falling-uterus

Ferretti, A., Caulo, M., Gratta, Del Gratta, C., Di Matteo, R., Merla, A., Montorsi, F., Pizzella, V., Pompa, P., Rigatti, P., Rossini, P. M., Salonia, A., Tartaro, A., \& Romani, G. L. (2005). Dynamics of male sexual arousal: Distinct components of brain activation revealed by fMRI. NeuroImage, 26, 1086-1096.

Holstege, G., Georgiadis, J. R., Paans, A. M. J., Meiners, L. C., van der Graaf, F. H. C. E., \& Reinders, A. A. T. S. (2003). Brain activation during human male ejaculation. The Journal of Neuroscience, 23 (27), 9185-9193.

Huynh, H. K., Willemsen, A. T. M., \& Holstege, G. (2013). Female orgasm but not male ejaculation activates the pituitary. A PET-neuro-imaging study. NeuroImage, 76, 178-182.

Komisaruk, B. R., Allen, K., Wise, N., Frangos, E., \& Birbano, W. (2013, November). Men's genital structures mapped on the sensory cortex: fMRI evidence. Poster presented at the meeting of the Society for Neuroscience, San Diego, California.
Komisaruk, B. R., Wise, N., Frangos, E., Liu, W., Allen, K., \& Brody, S. (2011). Women's clitoris, vagina, and cervix mapped on the sensory cortex: fMRI evidence. The Journal of Sexual Medicine, 8, 2822-2830.

Mastroianni, A. C., Faden, R., \& Federman, D. (1994). Women and health research. Ethical and legal issues of including women in clinical studies. Washington, DC: National Academies Press (US).

Mouras, H., Stoleru, S., Bittoun, J., Glutron, D., Pelegrini-Issac, M., Paradis, A.L., \& Burnod, Y. (2003). Brain processing of visual sexual stimuli in healthy men: A functional magnetic resonance imaging study. NeuroImage, 20, 855-869.

Moyer, M. W. (2010, July 28). Drug problem. Women aren't properly represented in scientific studies. Retrieved from http://www.slate.com/articles/health_an d_science/medical_examiner/2010/07/d rug_problem.html

Penfield, W. \& Boldrey, E. (1937). Somatic motor and sensory representation in the cerebral cortex of man as studied by electrical stimulation. Brain, 60, 389443.

Safron, A., Barch, B., Bailey, J. M., Gitelman, D. R., Parrish, T. B., Reber, P. J. (2007). Neural correlates of sexual arousal in homosexual and heterosexual men. Behavioral Neuroscience, 121 (2), 237248.

Wise, N. (2014). Genital stimulation, imagery, and orgasm in women. (Doctoral dissertation).

Retrieved from Rutgers, The State University of New Jersey Graduate School - Newark Electronic Theses and Dissertations http://dx.doi.org/doi:10.7282/T3X63PK $\mathrm{K}$

Woodward, T. L., Nowak, N. T., Balon, R., Tancer, M., \& Diamond, M. P. (2013). Brain activation patterns in women with acquired hypoactive sexual desire disorder and women with normal sexual function: A cross-sectional pilot study. Fertility and Sterility, 100 (4), 10681076 